\title{
UAV APPLICATION IN POST - SEISMIC ENVIRONMENT
}

\author{
V. Baiocchi ${ }^{\text {a }}$ D. Dominici ${ }^{\text {b }}$, M. Mormile ${ }^{\text {a }}$ \\ a DICEA - Area Geodesia e Geomatica, "Sapienza” University of Rome, \\ Via Eudossiana, 18-Zip, I-00184 Roma, Italy, \\ e-mail: valerio.baiocchi@uniroma1.it, \\ ${ }^{\mathrm{b}}$ Dept. Arc. and City Planning, University of L'Aquila, Via Gronchi 18, L’Aquila, 67100, Italy, \\ e-mail: donatella.dominici@univaq.it
}

KEY WORDS: UAV, earthquake, flight planning, L' Aquila, photogrammetry, UP23d

\begin{abstract}
:
On April 6, 2009, an earthquake of 6.3 magnitude struck central Italy with its epicentre near L'Aquila, at $42.3502^{\circ} \mathrm{N}, 13.3762^{\circ} \mathrm{E}$. The earthquake damaged 3,000 to 11,000 buildings in the medieval city of L'Aquila. Several buildings totally collapsed, 308 people were killed. The post emergency phase till now is just at its beginning step. Conventional surveying techniques using high precision total stations, GNSS receivers and laser scanners for investigations on damaged buildings are always becoming more automated, accurate and operative and even much more fast. Even if these techniques represent instruments of extreme operability there are still many evident limits on their use, especially regarding the survey of both the roofs and the facades of tall buildings or dangerous places, typical on post earthquake situations. So using micro UAVs for surveying in such particular cases, many of these problems can be easily bypassed. In fact, the present work aims on experimenting using multi-rotor micro UAVs, that will allow high quality image capturing on roofs and facades of structures in the old city center of L' Aquila. To obtain actual stereoscopic acquisitions of buildings some conditions on the geometry of acquisition have to be observed, for this reason, taking as a guideline classic flight photogrammetric, a flight planning software was developed. Accurate planning for UAVs acquisitions is very important also considering the reduced autonomy of such vehicles. This can be a strategic point if we want to use UAVs for early damage assessment and also for post event reconstruction planning.
\end{abstract}

\section{INTRODUCTION}

An earthquake strongly damaged L'Aquila, Italy, in April 2009. That earthquake, killed hundreds and left tens of thousands of inhabitants homeless, hitting the city's graceful and extensive historic centre, which was its cultural and economic heart. The main shock occurred at 3:32 local time (1:32 UTC) on 6 April 2009, and was rated 5.8 Richter magnitude and 6.3 on the moment magnitude; its epicentre was near L'Aquila, the capital of Abruzzo, which together with surrounding villages suffered most damage. The region affected by the seismic activity covers an ellipse-shaped area spanning about $15 \mathrm{~km}$ in length, parallel to the Apennines mountain chain and $5 \mathrm{~km}$ in wideness. According to the Italian National Institute for Geophysic and Vulcanology (INGV) this seismic activity is the result of a perpendicular fault movement on a NW-SE oriented structure which is part of the $800 \mathrm{~km}$ long segmented vertical fault system running all along the Apennines mountains and covering great number of Italy's most important monumental cities. Within this urban setting, the seismic event caused serious damage to numerous historic buildings, mainly including a valuable heritage that goes from the Baroque and Renaissance periods, including important churches and palaces. The first emergency stage took months to be completed and after it was evident that only a small step of the whole operation was done. Detailed surveying of all building is presently being performed using geomatic instruments as total stations, close range photogrammetry, laser scanners and deformation monitoring. Even if all these techniques can perfectly fulfil many crucial post hazard needs, there are still many cases where they show some limits. Most of the difficulties are related to the morphologic and architectural accessibility, problems typical of a post disaster scenario. Other methodologies like aerial photogrammetry are surely less dangerous but in most case produce are not enough detailed and accurate to study the damaged structures. On the other hand, using micro UAVs (Unmanned Aerial Vehicles) for surveying in such particular cases can easily bypass many of these problems (Eisenbeiss et al., 2010). For example there are no accessibility problems for an UAV mainly because of the extreme flying flexibility of these instruments that can permit the access of virtually any place (Eynard et al., 2010). These instruments are also fully or almost fully remotely controlled so they involve little risk for the operators simply because they can be far from the most dangerous area. It has to be considered that in historic centers, reconstruction has to be structurally planned trying to protect and conserve all the undamaged parts of the buildings reconstructing the damaged part in a way that will not create interference with the undamaged parts. So the structural planning of the reconstruction is a very complicated task that need a complete and accurate three-dimensional survey of the damaged buildings. From the ground this can be achieved with laser scanning surveys, but the higher parts of the buildings like the roofs cannot be acquired with a "classical" survey.

Also the possibility of flying in those low altitudes with such fast and easy repeatability of any survey provides very accurate results of high quality always in very economically convenient way.

\section{THE SOFTWARE "UP23d"}

The flight planning is a quite complex phase because it influence profoundly the results (Hrabar, 2008). A right photogrammetric survey, in fact, needs a correct number of high quality acquisitions with the correct geometry (Ioannis et al.,2003). To guarantee this quality the whole flight and thus the acquisition points have to be spatially distributed maintaining definite geometric characteristics as equal and correct air base and correct interaxes (Myungsoo, 2008). Considering as 
guideline, the case of a traditional photogrammetric airplane acquisition, it's well known that a flight is planned according to predetermined almost rectilinear paths parallel to each other; during the flight, images are taken in succession at regular time intervals, forming what is called a "swath"(Fig.1).

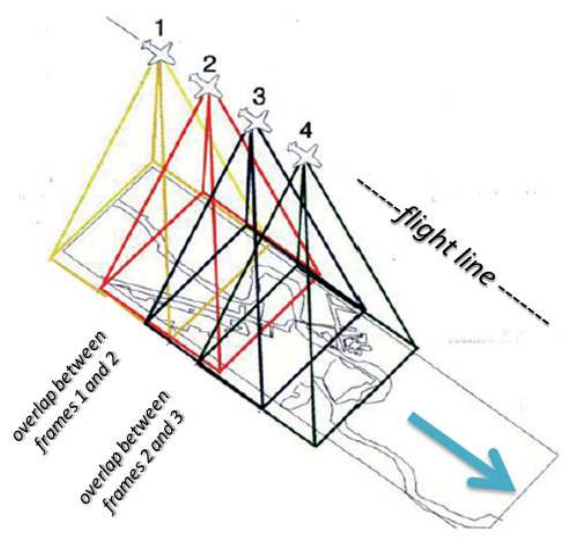

Figure 1. Case of a traditional photogrammetric airplane acquisition

Classical aerial stereoscopic acquisitions are constrained by a fixed ratio between flight height and length of air base. As it is well known an overlapping of 60 to $80 \%$ along the flight direction and 10 to $30 \%$ across the flight direction is usually considered as a guide line for aerial stereoscopic acquisitions (Kraus, 1994).

Currently planning is performed mainly based on the experience of the operators, for this reason we wanted to develop a software that can model the flight plan of the UAV to optimize acquisitions, evaluating at the same time if this approach is correct also for the multirotor UAVs using amateur or semimetric cameras (Barazzetti et al.,2011). The scope is to obtain all and only the frames needed to reconstruct the stereoscopy and to obtain geometrically correct reconstruction of the objects observed to reconstruct a DSMs (Chiabrando et al.,2011).

It was also observed that in urbanized areas like the historic centre of L'Aquila higher flight height can cause instabilities of the UAVs probably due to wind effects. Presently this problem is reduced taking three or more time the acquisitions needed, but this is obviously not efficient and also we observed that at lower heights buildings "cover" the UAVs from the effects of winds. Unfortunately lower heights means very little footprint and so higher number of acquisitions needed. Keeping all these problems in mind a scientific package UP23d will be illustrated that was realized to improve the optimization of the path obtaining a stereoscopic vision, considering all the mentioned constrains. This obviously using micro UAVs that seems to be the most flexible and presently easy to use, in a post seismic scenario.

The software "UP23d" (UAVs planner to 3 dimensional acquisitions) that we are developing, requires as input data the size, shape and position of the area to be acquired, the optic parameters of the camera installed on the drone and the desired final scale of the acquisitions. From these first input data the package suggest a flight altitude, which is proposed to the user which is free to edit if it's not suitable for the area to be investigated (for example, there is an obstacle with a height near to the one estimated).

Once estimated the flight height and consequently the final scale obtainable (hypnotized considering as guideline the classical aerophotogrammetric scale parameters), the values of longitudinal and across overlap have to be inserted in the software to allow the evaluation of the airbase needed to assure a correct stereoscopic reconstruction. After this step the software proceeds to the calculation of waypoints and footprint of each acquisition. As output the software returns the coordinates of the points where the UAVs has to stop to acquire the images; also a vector files of polygons that shows footprint of each frame is produced.

At its end the program provide a report file, which also contains the intermediate results that were estimated during its execution. Vector files can then be loaded into a GIS environment, as the open source Q-Gis, that allow to overlap to public access orthophoto satellite maps or to existing raster or vector cartography as shown in Fig. 2.

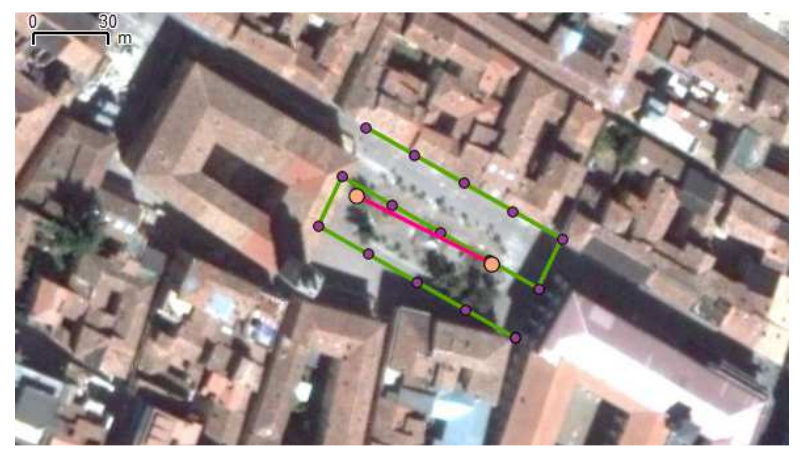

Figure 2. Vector files loaded into the open source software QGIS

In this figure, we can see that the vector files were produced by the software in the event of the experiment took place in "Piazza del Palazzo" in L'Aquila city.

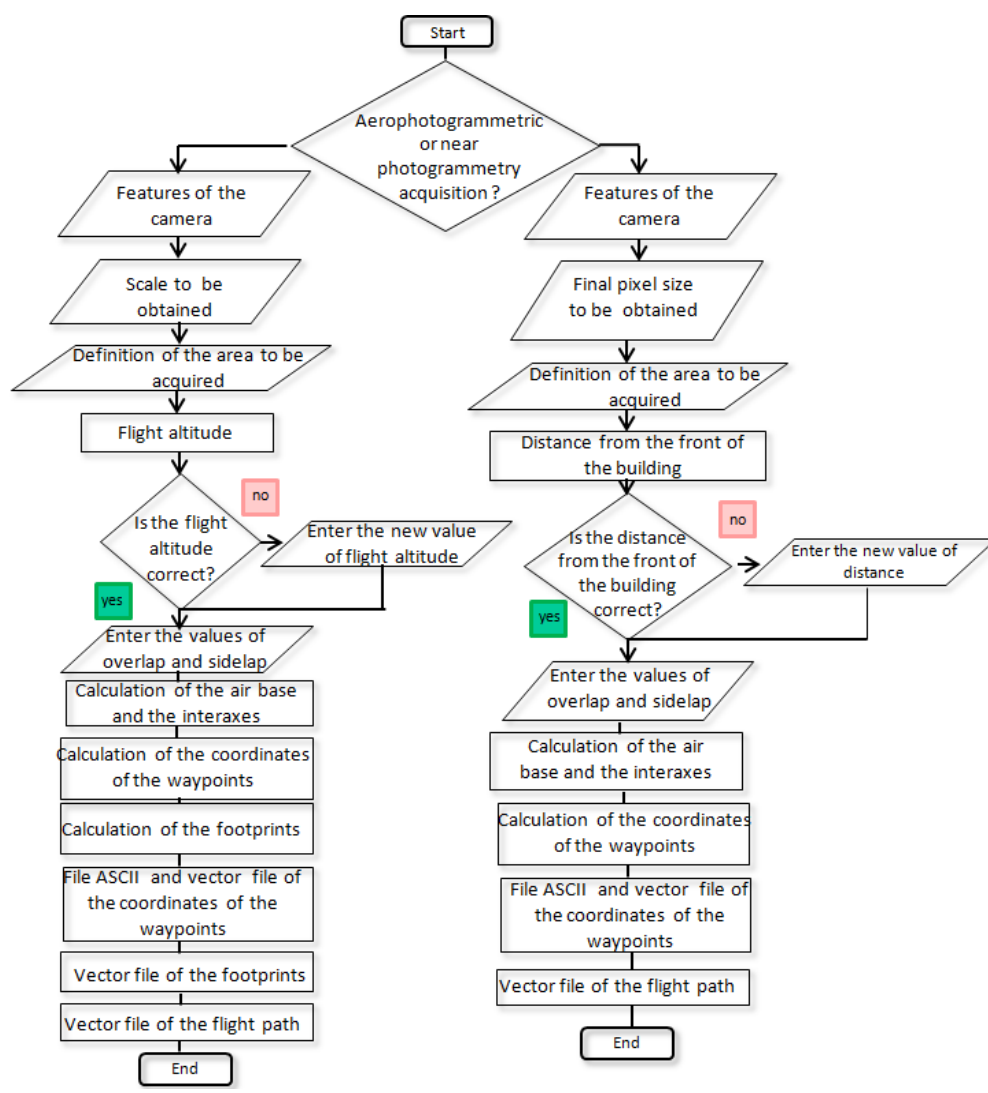

Figure 3. Flow chart of the software UP23d 
As the figure 2 shows, for the same parameters, the flight plan calculated by the software provides two acquisitions to reconstruct the stereoscopic of the area against fifteen acquisitions that were made by the UAV pilot. Carry out 15 acquisitions over an area of 60x40 meters extension are many, especially when you consider the limited autonomy of the micro UAVs.

In a post seismic environment UAVs can also be used to acquire facade of buildings for near photogrammetry acquisitions, in this case the package works in a slight different way as shown in figure 3 .

As for the aerophotogrammetric survey, as input data the size of the area to be acquired, the optic parameters of the camera installed on the drone and the dimension of the pixel in the acquisitions are needed.

\section{CASE STUDY}

To validate our approach a comparison with an actual UAV flight executed for near photogrammetry in L'Aquila was performed. In this first test the facade of "The Basilica di Santa Maria di Collemaggio", was acquired; the church is an important place of Catholic worship in the city of L'Aquila (Fig.4).

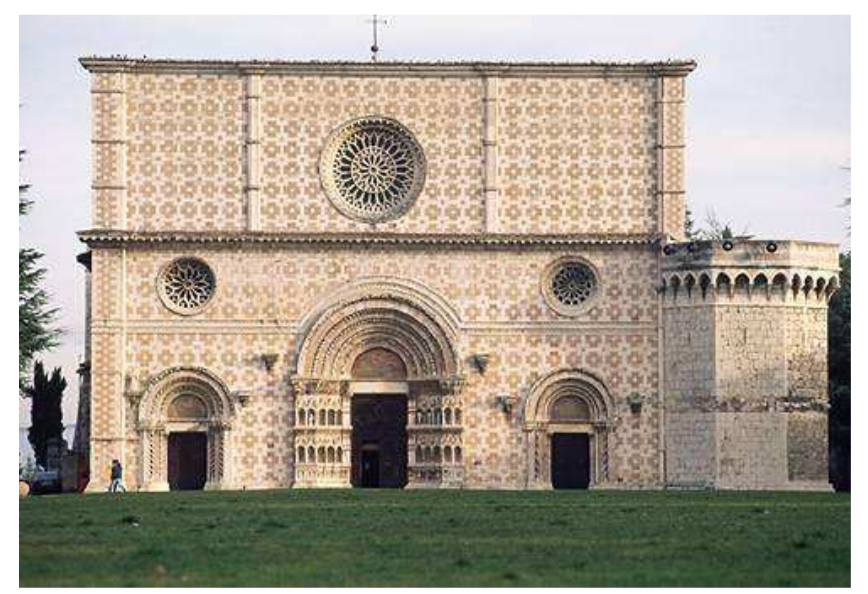

Figure 4. The Basilica di Santa Maria di Collemaggio

Established in 1287 by Pietro da Morrone, is the most important religious monument in the city in fact it's an UNESCO world heritage site. Contains the first Holy Door of the world and is home to a jubilee year unique of its kind. The basilica is the result of a complex blend of Romanesque architecture, the suction Gothic and Baroque style. During the earthquake of 2009, the Basilica was impressed so extremely serious. Part of the vault of the basilica collapsed causing the destruction of the terminal part of the nave. For this reason a complex work of restoration is going to be performed on the church.

This experimentation is the result of a collaboration between the Faculties of Engineering of L'Aquila university and "la Sapienza" university of Rome, and Aermatica company, that gently provided platform ANTEOS A2-MINI/B (Fig.5), including all on board sensors like optic commercial camera CANON S100 with focal length $5.2-26.0 \mathrm{~mm}(35 \mathrm{~mm}$ equivalent: $24-120 \mathrm{~mm}$ ), Infrared Camera Flir Tau320 and GPS navigation receivers to enable the automatic flight mode also with the necessary transmitters able to send all telemetry data in real time to the ground control station.

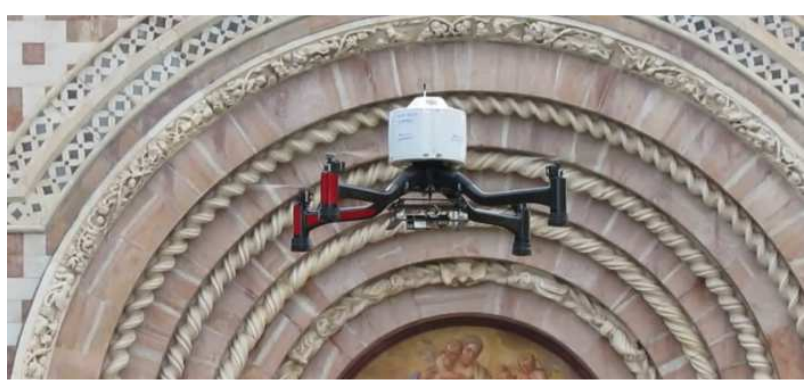

Figure 5. ANTEOS MINI during an acquisition

ANTEOS is a name of an RPA family, with four-rotor architecture and collective pitch control, positioned in "small" RPA range, having MTOW between 0 and $20 \mathrm{~kg}$. ANTEOS in "MINI" version, having $7 \mathrm{~kg}$ MTOW. This version have following features:

\begin{tabular}{|c|c|c|}
\hline Structure & Unit & ANTEOS “Mini” \\
\hline $\begin{array}{c}\text { External } \\
\text { Dimensions }\end{array}$ & -- & Molded carbon fiber \\
\hline Payload Capability & $\mathrm{Kg}$ & $1,25 \times 1,25 \times 0,55$ \\
\hline Autonomy & $\mathrm{min}$ & 2 \\
\hline $\begin{array}{c}\text { Automatic } \\
\text { Functions }\end{array}$ & -- & $\begin{array}{c}\text { Take-off, flight (and } \\
\text { hovering), landing, } \\
\text { safety procedures }\end{array}$ \\
\hline Navigation & -- & GPS + INS \\
\hline Pilot Mode & -- & Manual, Automatic \\
\hline $\begin{array}{c}\text { Operative Speed } \\
\text { Operative max. } \\
\text { height (restricted } \\
\text { by effective } \\
\text { regulations) }\end{array}$ & $\mathrm{Km} / \mathrm{h}$ & 25 \\
\hline $\begin{array}{c}\text { Operative max. } \\
\text { distance (restricted } \\
\text { by effective } \\
\text { regulations) }\end{array}$ & $\mathrm{m}$ & 50 \\
\hline $\begin{array}{c}\text { Motorization } \\
\text { Batteries }\end{array}$ & -- & Brushless \\
\hline & & Lipo Batteries \\
\hline
\end{tabular}

Table 6. Features of the ANTEOS MINI

For this reason, the choice of this platform was made trying to achieve excellent flying characteristics that can allow an optimum stability even in presence of wind or other non optimal climatic conditions and according to the payload specified by the manufacturer, which allows carrying all board sensors.

\section{COMPARISON OF AUTOMATED PLANNING WITH “CLASSICAL" PLANNING}

In this section we want to compare the UAV flight executed by the pilot, with the one planned by the software "UP23d". In order to make comparable the two flights, we provided as input to the software the same operating conditions under which the images were acquired during the survey at the Basilica. The flight plan designed by UP23d, included the following waypoints (Fig.7) expressed in UTM-WGS84 coordinates, which must be acquired at different flight altitude so as to obtain the values of overlap and sidelap desired. 


\begin{tabular}{|lll||}
\hline EST & NORD & FLIGHT ALTITUDE \\
368528.4454 & 4689099.3907 & 4.0000 \\
368526.0804 & 4689092.4763 & 4.0000 \\
368523.7154 & 4689085.5619 & 4.0000 \\
368521.3504 & 4689078.6474 & 4.0000 \\
368518.9855 & 4689071.7330 & 4.0000 \\
368516.6205 & 4689064.8186 & 4.0000 \\
368516.6205 & 4689064.8186 & 9.0000 \\
368518.9855 & 4689071.7330 & 9.0000 \\
368521.3504 & 4689078.6474 & 9.0000 \\
368523.7154 & 4689085.5619 & 9.0000 \\
368526.0804 & 4689092.4763 & 9.0000 \\
368528.4454 & 4689099.3907 & 9.0000 \\
368528.4454 & 4689099.3907 & 15.0000 \\
368526.0804 & 4689092.4763 & 15.0000 \\
368523.7154 & 4689085.5619 & 15.0000 \\
368521.3504 & 4689078.6474 & 15.0000 \\
368518.9855 & 4689071.7330 & 15.0000 \\
368516.6205 & 4689064.8186 & 15.0000 \\
368516.6205 & 4689064.8186 & 20.0000 \\
368518.9855 & 4689071.7330 & 20.0000 \\
368521.3504 & 4689078.6474 & 20.0000 \\
368523.7154 & 4689085.5619 & 20.0000 \\
368526.0804 & 4689092.4763 & 20.0000 \\
368528.4454 & 4689099.3907 & 20.0000 \\
368528.4454 & 4689099.3907 & 26.0000 \\
368526.0804 & 4689092.4763 & 26.0000 \\
368523.7154 & 4689085.5619 & 26.0000 \\
368521.3504 & 4689078.6474 & 26.0000 \\
368518.9855 & 4689071.7330 & 26.0000 \\
368516.6205 & 4689064.8186 & 26.0000 \\
4 & & \\
\hline & &
\end{tabular}

Figure 7. Waypoint coordinates calculated by the software UP23d

In figure 8 we can observe the difference between the actual acquisitions (light blue) and the planned acquisitions

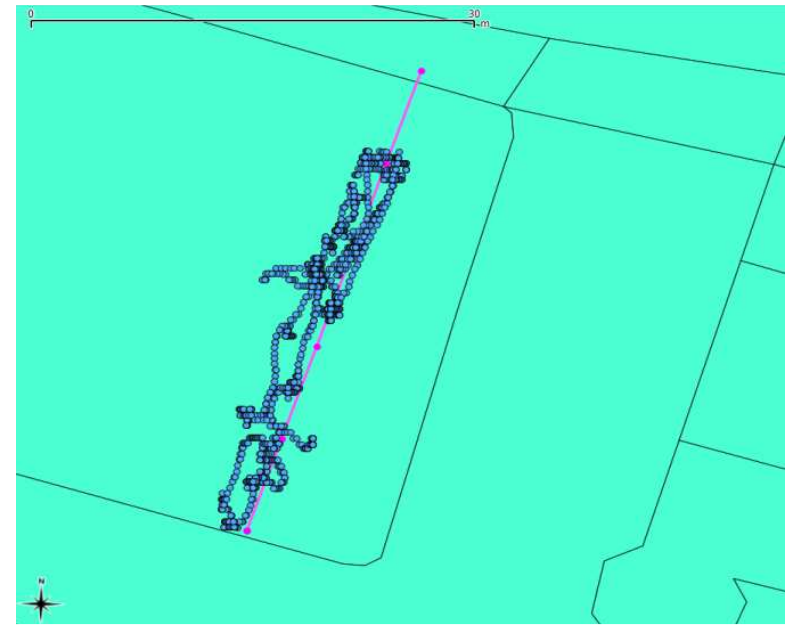

Figure 8. Comparison between the flight plan calculated by the software UP23d with that conducted by the UAV pilot

From this first analysis, we can note, that the number of acquisitions executed by the pilot was extremely redundant and this (as we explained) is presently the most diffused way to acquire.

To simulate an acquisition based on our planning software we looked for two acquisitions that simulate those calculated by the software UP23d. So we found some stereopairs that have nearly the same geometry planned by the software. (Fig.9)

In figure 9 we can observe two acquisitions that show similar geometry to one of the stereopair planned: they were acquired at lightly different flight altitudes, respectively, 18.9 and 18 meters, but this is correct for simulate an actual planned acquisition where the position of the UAV depends on the limited accuracy of the onboard GPS system. The tests for the extraction of DSM are presently in progress but first results seem encouraging (Fig.10).
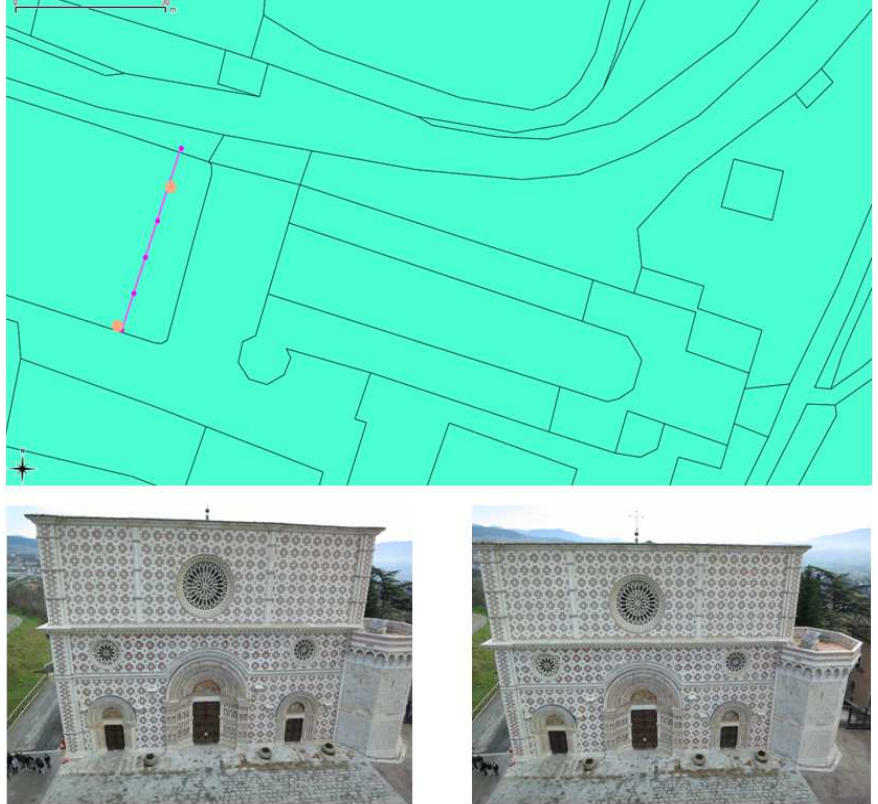

Figure 9. The stereopairs that have nearly the same geometry planned by the software
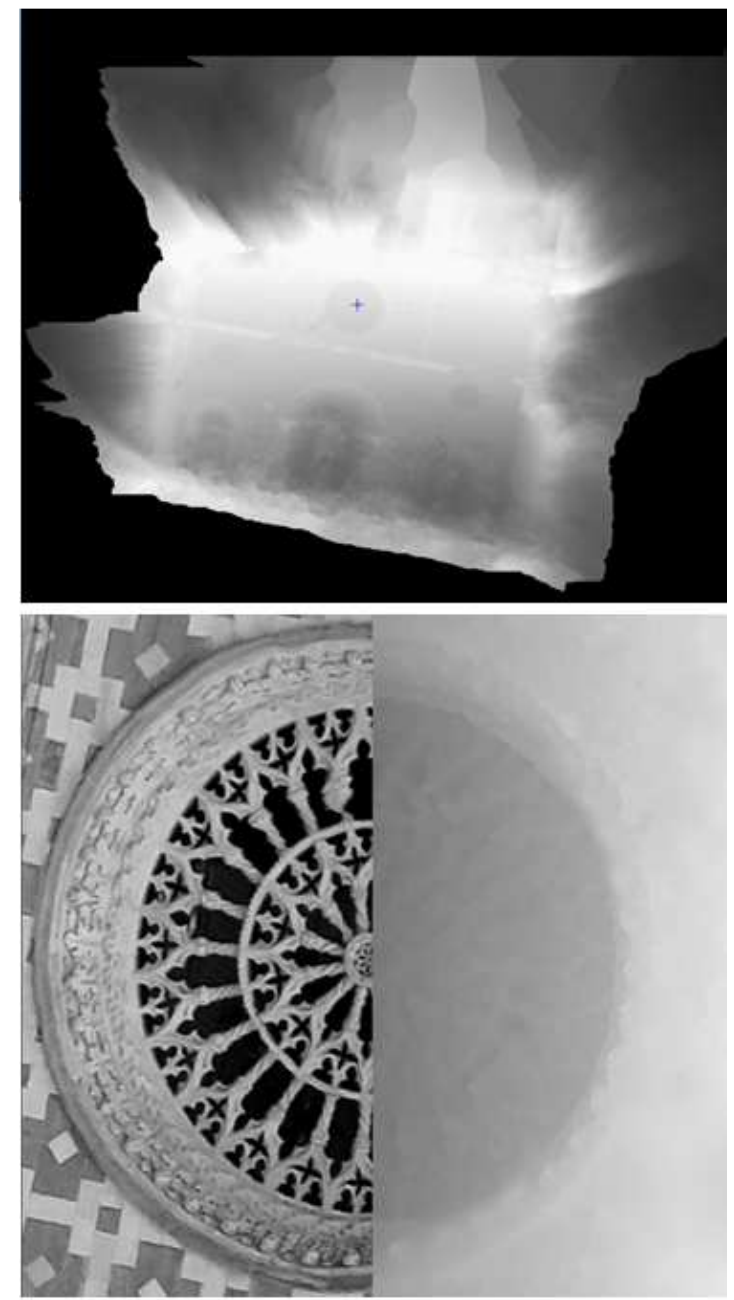

Figure 10. First test of DSM extraction from the stereopair 
Observing in detail the obtained DSM, we can see that a precision of one decimetre and in some cases also a centimetre is possible to be achieved. Presently it is not possible evaluate the accuracy because a laser scanning survey is in process.

\section{CONCLUSIONS AND FURTHER DEVELOPMENTS}

From these first tests it seems that the package UP23d correctly performs all the planned operations. Some tests of DSM extraction were performed and the precision appear to be comparable to other techniques. The possibility to export directly the flight plan in a GIS standard file like the shape format allow the complete interoperability of the software with all the other packages needed for an UAV flight.

Limits of this approach can be the limited accuracy of the GPS sensor on the UAV and the uncertainty of the correct acquisition of each frame that can be influenced by the instability of the UAV itself. Anyway the inaccurate acquisition (but only them) can be repeated with a predetermined flight plan with an obvious time saving.

When the planning will be completely optimized, the improved possibility of the UAV as operating time may disclose new possibilities of application as mapping of wider areas.

\section{REFERENCES}

1. Barazzetti L.,Nex F.,Remondino F.,Sarazzi D.,Scaioni M.(2011), "UAV photogrammetry for mapping and 3D modeling - current status and future perspectives ", International Archives of the Photogrammetry, Remote Sensing and Spatial Information Sciences, Vol. XXXVIII-1/C22 UAV-g, Conference on Unmanned Aerial Vehicle in Geomatics, Zurich, Switzerland (2011).

2. Chiabrando F., Nex F., Piatti D., Rinaudo F. "UAV and RPV systems for photogrammetric surveys in archaelogical areas: two tests in the Piedmont region (Italy)" Journal of Archaeological Science 38 $697 \mathrm{e} 710$ (2011).

3. Eisenbeiss H., Haarbrink R. B."Accurate DSM production from unmanned helicopter systems" ISPRS Technical Commission VII Symposium 100 Years ISPRS Advancing Remote Sensing Science, Vienna, Austria (2010).

4. Eynard D. and Vasseur P. and Demonceaux C. and Fremont V. "UAV Altitude Estimation by Mixed Stereoscopic Vision" The IEEE/RSJ International Conference on Intelligent Robots and Systems, 2010, Taipei, Taiwan (2010).

5. Hrabar S. "3D Path Planning and Stereo-based Obstacle Avoidance for Rotorcraft UAVs" 2008 IEEE/RSJ International Conference on Intelligent Robots and Systems Acropolis Convention Center Nice, France, (2008).

6. Ioannis K. Nikolos, Kimon P. Valavanis, Senior Member, IEEE, Nikos C. Tsourveloudis, and Anargyros N. Kostaras "Evolutionary Algorithm Based Offline/Online Path Planner for UAV Navigation" IEEE transactions on systems, man, and cybernetics-part b: cybernetics, vol. 33, no. 6, (2003).
7. Kraus K., 4th edition Italian translation Prof. Sergio DEQUAL - Politecnico di Torino Cap 3. Pag. 147 153(1994).

8. Myungsoo Jun "Path planning for unmanned aerial vehicles in uncertain and adversarial environments" In the Book Chapter of "Cooperative Control: Models, Applications and Algorithms", Kluwer, edited by S. Butenko, R. Murphey, and P. Pardalos (2008).

\section{ACKNOWLEDGEMENTS}

The authors thank the Aermatica company, that gently provided platform ANTEOS A2-MINI/B in Quad configuration, which made it possible to test. 